\title{
Performance Evaluation of Magnetic Resonance Images Coding Using Shape Adaptive Discrete Wavelet Transform
}

\author{
G.R.Suresh, S.Sudha and R.Sukanesh
}

\begin{abstract}
Region based coding is an important feature in today's image coding techniques as it follows different regions of the image will be encoded at different bit rates and hence at different qualities rather than encoding the entire image with a single quality constraints. This paper evaluates the features of using Shape Adaptive Wavelet Transform for the region based coding of the brain magnetic resonance images, in which the brain part will be encoded with more importance than the background. Shape Adaptive Discrete Wavelet Transform (SA-DWT) can transform the regions of interest and the background on the images independently and the coefficients can be encoded by using the SPIHT coding at different levels. This algorithm was compared with the existing wavelet based coding techniques and a better PSNR was achieved for the same bit rate by reconstructing the region of interest with high quality than the background.
\end{abstract}

Index Terms - Medical Image compression, Magnetic Resonance Images Coding, Shape adaptive wavelet transform, SPIHT coding.

\section{INTRODUCTION}

Recent advances in medical imaging technology lead to a tremendous increase in the use of volumetric medical images for diagnosis. As a result, huge volume of medical image data is generated in hospitals everyday and these needs to be stored for diagnosis and future follow up. Also in telemedicine, there is a need for the transmission of medical images for off-site consultation and diagnosis at faster rate with minimum bandwidth. Hence the compression of medical images plays a vital role for effective storage and transmission. Lossy compression schemes are not generally used for medical image compression so as to avoid any loss of clinically significant information, which may affect diagnosis. Region based compression can provide a better solution for this by coding the regions of interest with lossless coding and the background lossy. Such hybrid schemes can give better compression compared to the

Manuscript received January 15, 2009.

G. R. Suresh is with the Department of Electronics and Communication Engineering, Sona college of Technology, Salem - 5, TN, India. (phone: +91-96009-83735).

S. Sudha is also with the Department of Electronics and Communication Engineering, Sona college of Technology, Salem - 5, TN, India. (phone: +91-96009-83735).

R. Sukanesh is with the Department of Electronics and Communication Engineering, Thiagarajar college of Engineering, Madurai - 15, TN, India.. lossless schemes and also maintain the quality of the image.

Volumetric medical data are image sequences of any organ for the three dimensional view. These images are correlated both within and across slices to eliminate the redundancy. Some of the existing 3D compression techniques used motion compensation and encoded the image by the mesh formation. The deformation across the slices of the sequence is modeled as motion and the motion compensation algorithm is used to exploit the correlation between the slices [1]. This method can provide a lossless compression of the sequence but with increased expense of complexity. Region based compression methods based on wavelet transforms can perform better with relatively less complexity. In JPEG 2000 part - 1 [2], max-shift ROI coding is used which exploits intra band dependency and adopts EBCOT [3] algorithm for coding the bit planes of wavelet coefficients. In this, it is not necessary to send the shape information as side information for decoding but the coefficients will blend with the pixels at the boundary between the ROI and the background. In JPEG 2000 part- 2 [4], scaling based ROI coding is adopted whereby the coefficients associated with the ROI are scaled up by a certain number of bitshift and then the bit planes of the coefficients are encoded plane by plane.

An ROI coding technique combining shape-adaptive wavelet transform and scaling-based ROI is proposed in [5] in which, the samples within the object are transformed with shape-adaptive wavelet transform according to the shape-information and the samples within the object are scaled up by a certain number of bit-shifts, and encoded plane by plane. Ji-Zheng Xu et al.,[6] proposed a scheme for object based coding of video objects. Motion based 3D SA-DWT is used to transform the video objects and is coded with an entropy coder called Motion-based Embedded Sub-band Coding with Optimized Truncation Using (ESCOT), which takes advantage of the motion information and encodes each sub-band independently. This paper proposes a region based coding technique for the compression of volumetric magnetic resonance images using 3D SADWT for transformation and the coefficients are encoded using 3D SPIHT [7]. The performance of the proposed scheme is evaluated and compared with the existing schemes for its performance on magnetic resonance images.

\section{Shape AdAPtive WAVELET TRANSForm}

Shape Adaptive Discrete Wavelet Transform (SA-DWT) is an efficient algorithm for the transformation of arbitrary 
shaped object images [8]. Most important feature of the SA-DWT is that the number of coefficients after the transform will be same as the number of pixels in the original arbitrary shaped object in the image. It can well preserve the spatial correlation, locality and self-similarity between the sub bands. SA-DWT becomes ideal to conventional wavelet transform for a rectangular region or object. SADWT for three dimensional medical images is simply the extension of the 2D transform. The 2-D SA-DWT algorithm provides a way to efficiently decompose an arbitrarily shaped object into a multi-resolution object pyramid. The spatial correlation, locality, and object shape are well preserved throughout the SA-DWT. Thus, it enables multi-resolution coding of arbitrarily shaped objects. This method ensures that the number of coefficients to be coded in the transform domain is exactly the same as that in the image domain. The treatment of odd number of pixels in a segment ensures that there is not too much energy leaked into highpass bands in pyramid wavelet decomposition. Note that if the object is a rectangular image, the 2-D SADWT is identical to a standard 2-D wavelet transform.

The computational complexity of the SA-DWT depends on the number of pixels in the object. The computational complexity of the SA-DWT is no more than that of a conventional wavelet transform on a image. The overhead of the SA-DWT comes from searching segments in a row or column. Since this is a simple logic operation, it does not require too much in computational complexity. It increases the hardware design complexity, since new logic for handling the segment search is required. Similar to the conventional wavelet transform, the complexity of the SA-DWT depends on the number of filter taps. It could be simpler than the SA-DCT if the number of filter taps is few and could also be more complex than the SA-DCT if the wavelet filter has a large number of taps.

\section{Proposed Methodology}

The basic idea of the new 3D SA-DWT scheme is that the temporal decomposition is always performed along the motion trajectories. The corresponding pixels (1D array) obtained from motion estimation or other matching schemes are first aligned in the temporal direction. Then a 1D SA-DWT will be applied to this 1D array to obtain a 1D coefficient array. The coefficients in this 1D array will then be redistributed to their corresponding spatial position in each frame. After this a 2D transform will be applied on these frames.

\section{A. 3-D SADWT}

The steps mentioned below can perform the 3D SA-DWT, 1. Initialize:

Set $\mathrm{i}=0$, and mark all pixels within object boundary in all these $\mathrm{N}$ frames as UNSCANNED;

2. Form threads for temporal SA-DWT:

2.1. For every pixel pi(xi,yi) within object boundary in frame Pi,

2.1.1. If it is marked as UNSCANNED, then it becomes the first pixel of a new temporal thread. Let $\mathrm{j}=\mathrm{i}$;

2.1.2. If $p j(x j, y j)$ is a terminating pixel then $p j(x j, y j)$ is the last pixel of this temporal thread, go to 2.14. If pj(xj,yj) is not a terminating pixel and its corresponding pixel $\mathrm{pj}+1(x \mathrm{x}+1$, $y j+1)$ in frame $P j+1$ is marked as UNSCANNED, where $(x j+1, y j+1)=(x+m v x, y+m v y)$ and $(m v x$, mvy $)$ is the motion vector from pixel $\mathrm{pj}(\mathrm{xj}, \mathrm{yj})$ in frame $\mathrm{Pj}$ to its corresponding pixel $p j+1(x j+1, y j+1)$ in frame $P j+1$, then $p j+1(x j+1, y j+1)$ is added as the next pixel in this temporal thread and is marked as SCANNED. If the corresponding pixel in the next frame is marked as SCANNED, then this temporal thread terminates at this pixel.

$$
\text { 2.1.3. } \mathrm{j}=\mathrm{j}+1 \text {, if } \mathrm{j}<\mathrm{N} \text { go to } 2.1 .2
$$

2.1.4. Perform $1 \mathrm{D}$ arbitrary length wavelet filtering for this temporal thread: Pk (xk,yk), k=i,..,j-1 and obtain a transformed low-pass thread: Lk(xk,yk), $\mathrm{k}=\mathrm{i}, \ldots, \mathrm{j}-1$; and high-pass thread $\mathrm{Hk}(\mathrm{xk}, \mathrm{yk}), \mathrm{k}=\mathrm{i}, \ldots, \mathrm{j}-1$.

These low-pass coefficients $\mathrm{Lk}(\mathrm{xk}, \mathrm{yk})$ are placed into the low-pass frame $\mathrm{k}$ at position (xk,yk). And these high-pass coefficients $\mathrm{Hk}(\mathrm{xk}, \mathrm{yk})$ are placed into the high-pass frame $\mathrm{k}$ at position $(\mathrm{xk}, \mathrm{yk})$.

Isolated pixels can be scaled by sqrt (2) and put back into their corresponding positions in both

low-pass and high-pass frames.

3. $\mathrm{i}=\mathrm{i}+1$, if $\mathrm{i}<\mathrm{N}$ go to 2.1

4. Subsample low-pass frames at even frames to obtain temporal low-pass frames and subsample highpass frames at odd frames to obtain temporal high-pass frames.

5. If more temporal decomposition levels are needed, repeat step 1-4 for the low-pass frames. Note that the motion vectors from frame $\mathrm{Pk}$ to $\mathrm{Pk}+2$ can be obtained by adding the motion vectors from $\mathrm{Pk}$ to $\mathrm{Pk}+1$ and $\mathrm{Pk}+1$ to $\mathrm{Pk}+2$.

6. Perform spatial 2D SA-DWT transforms according to their spatial shapes for every temporally transformed frame.

The above SA-DWT algorithm provides a way to efficiently decompose an arbitrarily shaped object into a multi-resolution object pyramid. The spatial correlation, locality, and object shape are well preserved throughout the SA-DWT. Thus, it enables multi-resolution coding of arbitrarily shaped objects. This method ensures that the number of coefficients to be coded in the transform domain is exactly the same as that in the image domain. The treatment of odd number of pixels in a segment ensures that there is not too much energy leaked into high-pass bands in pyramid wavelet decomposition. Note that if the object is a rectangular image, the 2-D SA-DWT is identical to a standard 2-D wavelet transform.

The SA-DWT can be implemented as line-based wavelet transform as described above. Thus, the working buffer needed is no larger than the larger of width and height of the region of interest. In order to store the original image and the transformed coefficients, there needs to be a main memory of $\mathrm{W}$ X $\mathrm{H}$ words, where $\mathrm{W}$ and $\mathrm{H}$ are width and height of the region of interest, respectively. The current implementation of SA-DWT is performed using separable 1-D wavelet transforms, that is, each level of decomposition needs to read and write the memory for that subband twice. The computational complexity of the SA-DWT depends on the number of pixels in the ROI. If the ROI of the image has a width $\mathrm{W}$ and a height $\mathrm{H}$, the computational complexity of the SA-DWT is no more than that of a conventional wavelet $-115$ 
transform on a $W X H$ image. The overhead of the SA-DWT comes from searching segments in a row or column.

\section{B. $3 D$ SPIHT}

The main objective in applying three-dimensional coding schemes is to exploit the correlations between image pixels across the third dimension (inter-slice or temporal direction) to achieve better compression ratio. 3D SPIHT coding scheme is extended from 2D SPIHT algorithm [9]. 3D dataset is decomposed using three-dimensional Shape adaptive wavelet transform into three dimensional sub bands, then SPIHT coding algorithm is used to create compressed bit stream using bit plane coding. 3D SPIHT decoder extracts information from the bit stream to create the reconstructed image sequence. Wavelet transform on three-dimensional data can be carried out by calculating one-dimensional transform along the temporal dimension and then applying the two-dimensional spatial transform on each resulting frame. At the next decomposition level, wavelet transform is applied on the lowest sub band and this process repeated in a cascaded manner, until desired number of levels is achieved. Alternatively 3D wavelet transform can be calculated by first performing one dimensional transform along the temporal dimension until the required number of temporal decomposition levels is obtained and then carrying out the two dimensional transform on each temporal-transformed frame up to the desired number of spatial decomposition levels.

The representations for multiple volumetric regions constitute the conventional wavelet coefficient volume. The spatio-temporal orientation trees can be constructed by using the conventional SPIHT coding algorithm. However, when coefficients are to be encoded, an important issue is that there exist coefficients from different volumetric regions within the same spatio-temporal orientation tree. This problem can be solved by using different labels, which help to distinguish the coefficients from different volumetric regions. For encoding of the transform coefficients pertaining to a region, we partition all the spatiotemporal orientation trees using the conventional method and encode the information about wavelet coefficients in a particular volumetric region. This scheme directly extends the original algorithm to encode multiple regions at multiple quality constraints or at a specified quality constraint. Thus, independent bitstreams for each volumetric region are produced and a context-based arithmetic-coding algorithm can then be used to improve coding efficiency by exploiting the dependencies between significant information of wavelet coefficients.

\section{EXPERIMENTAL RESULTS AND DISCUSSION}

For experimental verification on the performance of the proposed scheme, we use MR brain volumetric dataset (256x256x60 at 8bits) collected from the MRI scan center. The data set consists of 60 frames and each with a size of 256 X 256 stored as DICOM images.

The performance of the proposed algorithm with shape adaptive wavelet transform and 3D SPIHT is evaluated using an MRI dataset of 60 frames which is considered as two groups with each group contain 30 frames. We have tested the performance using different types of wavelet filters and observed that bi-orthogonal filters can give good PSNR while compared with that of other wavelet filters. The graph in figure 2 shows the PSNR obtained for different frames of the data set for the first group of 30 frames for the bitrate of 1 BPP and figure 3 shows the average PSNR obtained while coding the coefficients with different bitrates from 0.1 to $1 \mathrm{bpp}$. Figure 4 shows the original and reconstructed image of the 30th frame encoded using the proposed algorithm with a bit rate of 1bpp. This shows that the images can be reconstructed without much loss in the region of interest.

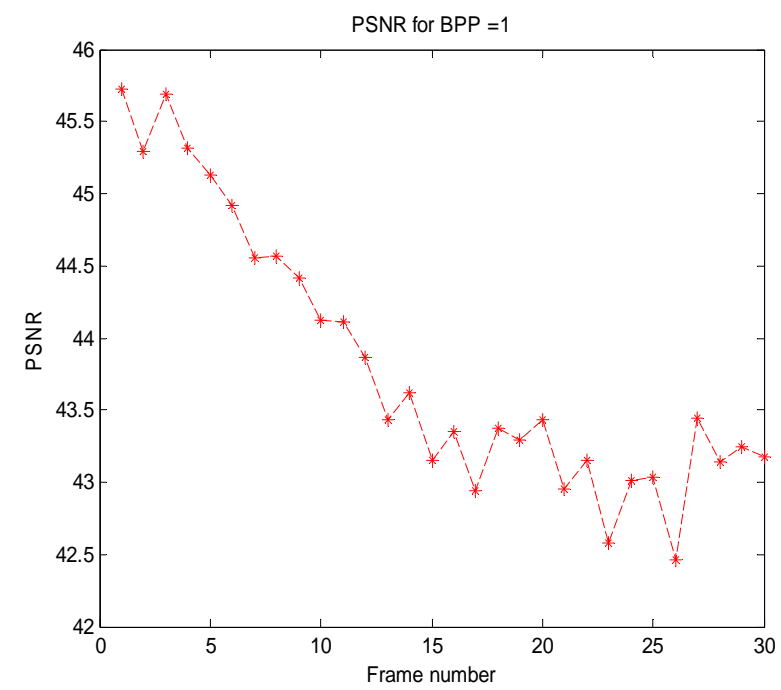

Figure 2: Plot on the PSNR values of different frames reconstructed from the proposed coding scheme.

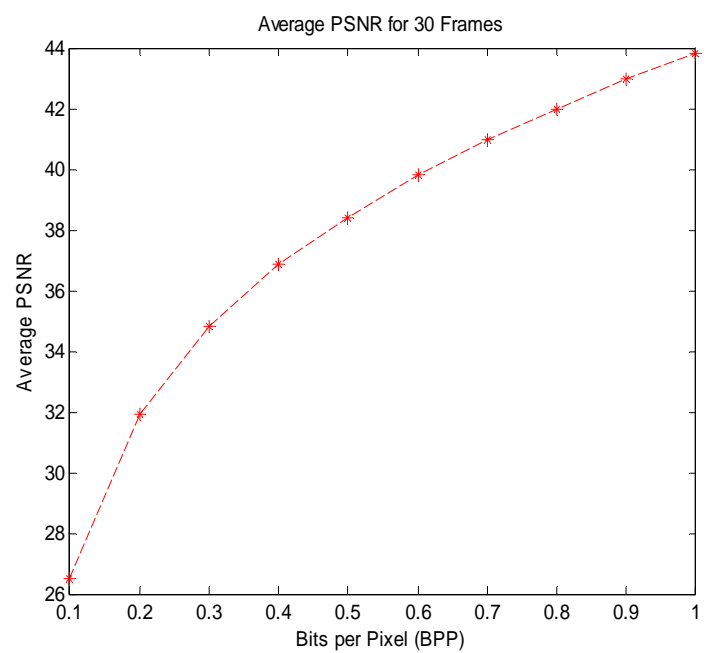

Figure 3: Plot on the average PSNR values obtained for different bit rates 


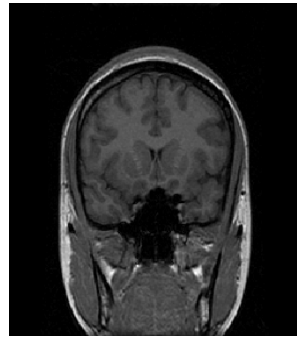

(a)

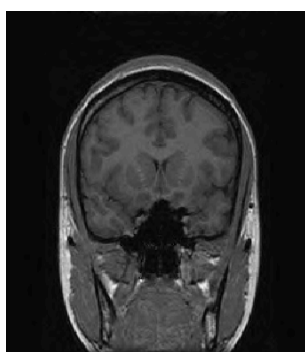

(b)

Figure 4: (a) Original Image of the 30th frame of the MRI sequence, (b) Reconstructed Image encoded with a bit rate of $0.8 \mathrm{bpp}$.

\begin{tabular}{|l|l|l|l|l|l|l|}
\hline \multirow{2}{*}{$\begin{array}{l}\text { Size } \\
\text { of the } \\
\text { group } \\
\text { of } \\
\text { Slices }\end{array}$} & \multicolumn{4}{|l}{\begin{tabular}{l}
\multicolumn{4}{l}{ Average PSNR in dB } \\
\cline { 2 - 7 }
\end{tabular}} & $\begin{array}{l}\text { Proposed Scheme } \\
\text { SADWT-SPIHT) }\end{array}$ & \multicolumn{2}{l}{ WP-SPIHT } & \multicolumn{2}{l|}{ DY-SPIHT } \\
\hline & 0.8 & $1 \mathrm{bpp}$ & 0.8 & $1 \mathrm{bpp}$ & 0.8 & $1 \mathrm{bpp}$ \\
\hline 15 & 41.87 & 43.76 & 39.36 & 41.52 & 38.26 & 40.27 \\
\hline 30 & 41.98 & 43.82 & 39.56 & 41.68 & 38.43 & 40.46 \\
\hline 60 & 42.03 & 43.93 & 39.73 & 41.72 & 38.46 & 40.83 \\
\hline
\end{tabular}

TABLE 1: COMPARISON OF THE PERFORMANCE OF THE PROPOSED ALGORITHM WITH THE EXISTING METHODS FOR A BIT RATE OF 0.8BPP AND $1 \mathrm{BPP}$

The rate-distortion performance of the proposed algorithm is also compared with other methods which are based on conventional wavelet transforms and SPIHT coding like dyadic-wavelet SPIHT (DY-SPIHT), Wavelet Packet SPIHT (WP-SPIHT) for the compression of MRI volumetric datasets. The performance of different coding schemes is measured in terms of its PSNR and is given in the table 1. Figure 4 shows the plot on the performance of these coding schemes for different bit rates from $0.1 \mathrm{bpp}$ to $1 \mathrm{bpp}$.

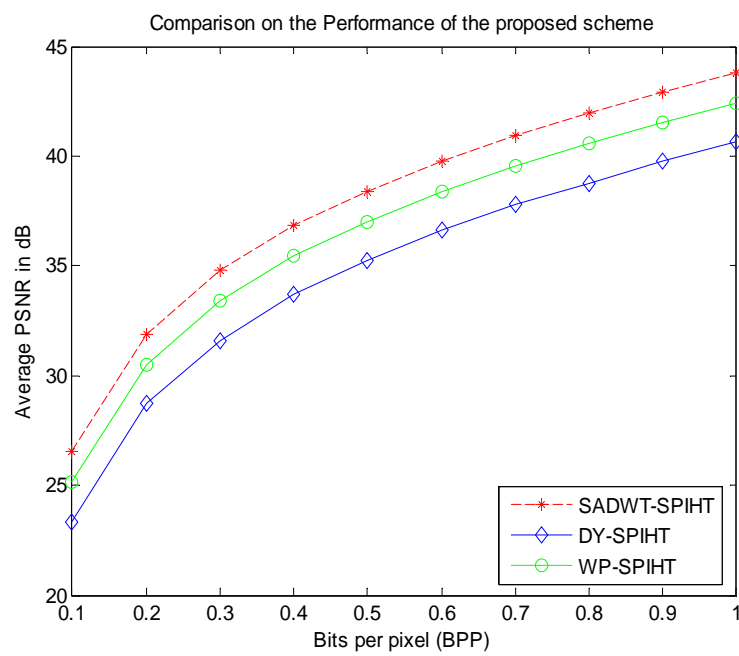

Figure 5: Plot on comparative performance of the proposed algorithm with other conventional coding schemes

The results show that the proposed scheme can perform better than existing wavelet based coding schemes by an average of $1 \mathrm{~dB}$ better than wavelet packet SPIHT coding and nearly $2 \mathrm{~dB}$ better than conventional wavelet SPIHT coding for the bit rate of $0.8 \mathrm{bpp}$. Also the proposed algorithm can have better efficiency for higher group of slices.

\section{CONCLUSION}

In this paper, the novelty of using Shape adaptive wavelet transform for the region based compression of volumetric MR Images is discussed. This approach can perform better than the schemes which are using conventional wavelet transforms. The salient feature of the SADWT helps to encode the pixels in the region of interest to be encoded losslessly and the background with less importance. In this scheme, $90 \%$ of the bit budget is used to code the ROI, which can reconstruct the images with high quality. Since other 3D coding algorithms do not support encoding of multiple ROIs, we compare the R-D performance of the scheme with the existing conventional SPIHT. The experimental results show that the proposed scheme provides a better PSNR when compared to other existing volumetric medical data compression techniques.

\section{REFERENCES}

[1] R. Srikanth and A. G. Ramakrishnan, "Contextual Encoding in Uniform and Adaptive Mesh-Based Lossless Compression of MR Images", IEEE Transactions on Medical Imaging, Vol. 24, No. 9, Sep 2005 .

[2] ISO/IEC 15444-1, JPEG2000 Image Coding System.

[3] D. Taubman, "High performance scalable image compression with EBCOT," IEEE Trans. Image Process., vol. 9, no. 5, pp. 1158-1170, May 1997

[4] ISO/IEC 15444-2, JPEG2000 Image Coding System: Extensions.

[5] Ikuro Uenoa, William A. Pearlman "Region of interest coding in volumetric images with shape-adaptive wavelet transform",SPIE/IS\&T Electronic Imaging2003, Proceedings SPIE vol.5022,Jan 2003.

[6] Ji-Zheng Xu, Shipeng Li and Ya-Qin Zhang, "Three-dimensional shape-adaptive discrete wavelet transforms for efficient object-based video coding", IEEE/SPIE Visual Communications and Image Processing (VCIP) 2000, Perth, June 2000.

[7] B-J. Kim and W.A. Pearlman, "An Embedded Wavelet Video Coder Using Three-Dimensional Set Partitioning in Hierarchical Trees," IEEE Data Compression Conference, pp. 251-260, March 1997.

[8] S.Li and W.Li, "Shape-adaptive discrete wavelet transform for arbitrary shaped visual object coding," IEEE Trans. Circuits Syst. Video Technol., vol. 10, pp.725-743, 2000

[9] A. Said and W.A. Pearlman, "A New Fast and Efficient Image Codec Based on Set Partitioning in Hierarchical Trees, "IEEE Trans. Circuits Syst. Video Technol., vol. 6., pp. 243--250, June 1996.

[10] Li-bao Zhang and Xian-chuan Yu, "New Region of Interest Image Coding Using General Layered Bitplane Shift for Medical Image Compression" International Journal of Computational Intelligence Research, Vol.3, No. 1, pp. 97-104, 2007.

[11] Tasdoken. Y, and A. Cuhadar, "ROI coding with region-based integer wavelet transforms and unbalanced spatial orientation trees", Proceedings of the SPIE Conference on Image and Video Communications and Processing, 2003. 
G.R. Suresh received his B.E degree in Electronics and Communication Engineering from Manomaniam Sundaranar University, Tamil Nadu, India, in 1997 and M.E degree in Communication Systems in Thiyagarajar College og Engineering ,Madurai, Tamil Nadu, India, in 2000 . He is currently pursuing Doctoral programme under the guidance of Dr.(Mrs.)R.Sukanesh in Medical Imaging at Anna University Chennai. He is working as Assistant Professor in Department of Electronics and Communication Engineering at Sona College of Engineering \& Technology, Salem, India. He has 12 years of teaching experience at college level. He has published eight papers in International, national journals. His area of interest includes image processing, Signal processing, VLSI,. He is a member in IEEE society and life member in ISTE.

S. Sudha. received her B.E degree in Electronics and Communication Engineering from Madurai Kamaraj University, Tamil Nadu, India, in 1996 and M.E degree in Applied Electronics from Madurai Kamaraj University, Tamil Nadu, India, in 2000 . She is currently pursuing Ph.D degree under the guidance of Dr.(Mrs.)R.Sukanesh in Medical Imaging at Anna University Chennai. She is working as Assistant Professor in Department of Electronics and Communication Engineering at Sona College of Engineering \& Technology, Salem, India. She has 12 years of teaching experience at college level. She has published eight papers in International, national journals.Her area of interest includes image processing, Signal processing. She is a life member of ISTE.

Dr.(Mrs)R. Sukanesh, Professor in Biomedical Engineering has received her bachelor's degree from Government college of Technology, Coimbatore in 1982. She obtained her M.E (Communication systems) degree from PSG College of Technology, Coimbatore in 1985and Doctoral degree in Bio-Medical Engineering from Madurai Kamaraj University, Madurai in 1999.Since 1985 she is working as a faculty in the Department of Electronics and Communication Engineering at Thiyagarajar College of Engineering, Madurai, and presently she is the Professor of ECE , and Heads the Medical Electronics division in the same college. Her main research areas include Neural networks , Bio-signal processing and mobile communication. She is guiding Twelve $\mathrm{PhD}$ candidates in these areas..She has published several papers in national, International journals and also published around eighty papers in National ,International conferences both in India and abroad. She has delivered number of invited lectures in various universities. She has a Diplamo in Higher learning and has co-authored a book on Gandhian thoughts. She is a reviewer for International journal of Biomedical sciences and International Journal of Signal Processing .She is an Editorial board member for Journal of Engineering studies. She contributed a chapter titled," Impact of Information Technology business, edited by Prof Biswajeet Pattnayak. She is the recipient of The President of India's Prize (English)-2006 and The Jawaharlal Nehru memorial Prize-2006 from IE(India) for the paper entitled "Genetic Algorithm optimization of fuzzy outputs for classification of epilepsy risk level from EEG". She is fellow of Institutions of Engineers (India).She is a life member of Bio-medical Society of India, Indian Association of Bio-Medical Scientists and ISTE. 